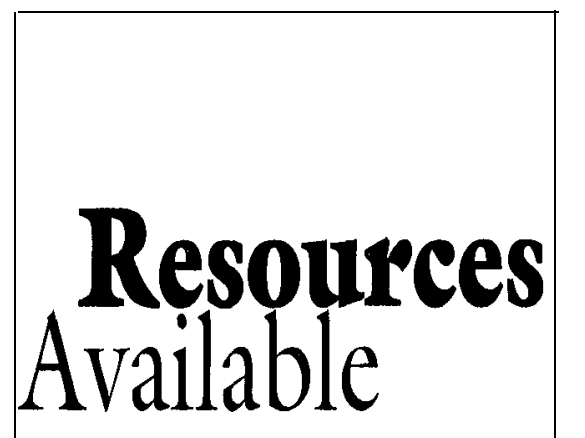

\section{Training and Pruning Apple and Pear Trees}

C.G. Forshey, D.C. Elfving; and Robert L. Stebbins, American Society for Horticultural Science, 113 South West St., Suite 400, Alexandria, VA 22314-2824. 1992. 166 pp. $\$ 30.00$

The authors briefly review the physiological effects of pruning and describe basic pruning cuts. They discuss when to prune, pruning practices, and their effect on the tree. The pruning manual presents general guidelines for training young trees and the objectives ofdifferent training and management systems relative to desired tree size and tree density. While the publication is limited to the moreextensively used higher tree-density systems, the authors acknowledge that innovative orchardists and researchers continue to develop various versions and modifications of these systems.

The manual is illustrated with 77 photographs and drawings of training and pruning concepts. The book will be useful for basic pomology courses, extension agents, and orchardists or individuals seeking pruning guidance.

Jerome Hull, Jr. Dept. of Horticulture Michigan State Univ. East Lansing; MI 48324

\section{Irrigation Scheduling Programs for Cabbage and Zucchini Squash}

James E. Ells, Dept. of Horticulture, Colorado State Univ., Fort Collins, CO 80523. Send a pre-fomatted computer diskette (3 or 5.25 inch) onto which the program will be copied and the diskette returned to the sender.

A detailed description of the programs is on pp. $448-453$ of this issue of HortTechnology. This program operates on IBM-compatible computers and requires the input of wind, radiation, temperature, humidity, and precipitation to determine the time and amount of irrigation to be applied.

\section{Non-circulating Hydroponic Methods}

Videotape. Produced by Bernie Kratky, Sheldon Furutani, and The UHH Media Center. The University of Hawaii-Hilo, College of Agriculture, 200 W. Kawili St, Hilo, HI 96720-4091. $\$ 50.00$

Non-circulating hydroponic methods can be simple, inexpensive, require low maintenance, and they do not require electrical power and complex equipment. Yet, yields from plants growing by these methods can be as good as those from conventional soil culture.

This 38-min video features Univ. of Hawaii-Hilo student projects wherein beans, cucumbers, and lettuce are grown by various hydroponic methods.

Two students successfully grew crops by conventional aerated and circulating hydroponic systems, but they explained that these systems required electrical power and pumps. The other students proudly displayed their simpler, non-circulating hydroponic systems. Sub-irrigation, non-circulating hydroponic projects consisted of partially immersing a hapuu $\log$ or a pot or a recycled beverage can into a stagnant tank of nutrient solution. One student showed how a screen can be placed between the nutrient solution and the tank top cover to encourage root growth. Another student demonstrated that the lower portion of a forestry tube may be immersed in a tank of nutrient solution and the resultant nutrient solution uptake by capillary action eliminated the need for additional fertilization or watering from transplanting until harvest. Several of these hydroponic methods were used to grow crops above a fish tank.

An historical background and the concepts of non-circulating hydroponics also are discussed. Growers, Master Gardeners, and teachers will find this to be an easy-to-understand introduction into the world of noncirculating hydroponics. 